\title{
Gestión universitaria de la innovación social promovida desde espacios académicos relacionados con el emprendimiento, la investigación y la proyección social
}

\author{
University management of social innovation promoted from academic spaces \\ related to entrepreneurship, research and social projection
}

\author{
Irina Margarita Jurado-Paz' \\ Michael Alexander Morán-Vallejo²
}

Recibido: julio 23 de 2018

Aceptado: diciembre 22 de 2018

\section{Resumen}

El presente artículo expone el diagnóstico del Programa de Internado Rural Interdisciplinario, PIRI, adelantado en una institución universitaria colombiana, con el fin de identificar elementos para mejorar su implementación. La investigación es de carácter descriptivo, con enfoque mixto, donde se emplearon encuestas y entrevistas para recolectar la información. La muestra fue de 214 estudiantes, pertenecientes a diversos programas de la Corporación Universitaria Autónoma de Nariño. Los resultados indican aportes significativos de PIRI, tanto a las instituciones como a los estudiantes que han participado del programa. PIRI se constituyó en un espacio educativo para que los estudiantes desarrollen competencias y apliquen sus conocimientos en temas relacionados con la innovación social. Sin embargo, existen dificultades como el bajo número de participantes, junto con la carencia de procedimientos claros que permitan una adecuada articulación de las entidades vinculadas a PIRI. En este sentido, se formuló un modelo que facilite la gestión universitaria de la innovación social en la institución objeto de estudio, que articula las dependencias de emprendimiento, investigación y proyección social, para que los beneficios se orienten tanto a la comunidad universitaria como a los territorios.

Palabras clave: innovación social, gestión universitaria, responsabilidad social universitaria, emprendimiento.

\begin{abstract}
This article shows the diagnosis of the Interdisciplinary Rural Internship Program, PIRI, held in a university institution in order to identify elements to improve its implementation. The research is descriptive, with a mixed approach, where surveys and interviews were used to collect information. A sample of 214 students was considered, belonging to different programs of the Autonomous University Corporation of Nariño. The results indicate significant contributions from PIRI to the institutions and to the students who have participated in the program. PIRI is used in an educational space for students to develop skills and apply their knowledge on issues related to social innovation. However, there are also difficulties such as the low number of participants, together with the lack of clear procedures that allow an adequate articulation of the entities linked to PIRI. In this sense, a model was formulated to facilitate the university management of social innovation in the institution under study, which articulates the dependencies of entrepreneurship, research and social projection, so that the benefits are oriented both to the university community and to the territories.
\end{abstract}

Keywords: social innovation, university management, university social responsibility, entrepreneurship.

1 Administradora de Empresas, Magíster en Administración de Empresas, Corporación Universitaria Autónoma de Nariño, Pasto, Colombia. E-mail: irina.jurado@aunar.edu.co

2 Administrador de empresas, Corporación Universitaria Autónoma de Nariño, Pasto, Colombia. E-mail: miki.9301@gmail.com 


\section{Introducción}

Las organizaciones empresariales, en especial las que tienen un enfoque hacia el desarrollo social, tienen hoy un gran desafío puesto que más allá de ser económicamente rentables, deben también contribuir responsablemente con el crecimiento social y generar procesos que sean amigables con el ambiente (Cardona-Arbeláez, Alzate-Alvarán \& Lora-Guzmán, 2018; Rodríguez \& Alvarado, 2008). En este sentido, es preciso referenciar a la Comisión de las Comunidades Europeas (2001), que define la responsabilidad social como: "un concepto con arreglo al cual las empresas deciden voluntariamente contribuir al logro de una sociedad mejor y un medio ambiente más limpio".

Las Instituciones de Educación Superior, IES, no pueden quedarse al margen de esta tendencia organizacional; más aún cuando varias de las dimensiones de este tipo de instituciones se relacionan con la proyección hacia la comunidad, enmarcada en unos criterios de respeto, compromiso y aporte significativo para un crecimiento con equilibrio social (Del Río-Cortina, Cardona-Arbeláez \& Guacarí-Villalba, 2017). Esta relación de la universidad con su entorno es concebida de diferentes maneras, entre ellas se encuentra una que permite la articulación de la docencia y la investigación (Lópezde Parra, Polanco-Perdomo \& Correa-Cruz, 2017), siendo este punto el eje de partida sobre el que se sustenta el presente documento.

La Corporación Universitaria Autónoma de Nariño, AUNAR, en su deseo de trabajar en pro del desarrollo territorial, generó un proceso articulado con la Corporación Escuelas del Sur, ES, organización sin ánimo de lucro con presencia en el territorio nariñense desde 2014. Las dos entidades firmaron un convenio de cooperación interinstitucional que ha permitido la realización del Programa de Internado Rural Interdisciplinario, PIRI, en el municipio de Pasto entre julio de 2015 y diciembre de 2017; en el se han trabajado líneas de acción a través de la investigación aplicada, la innovación y la proyección social. El programa ha contado con la participación de la comunidad estudiantil universitaria, la Alcaldía Municipal de Pasto, el Programa de las Naciones Unidas para el Desarrollo (PENUD), algunas organizaciones locales de la población beneficiaria y otras instituciones de educación superior presentes en la ciudad.

Para AUNAR, el ejercicio del PIRI ha generado importantes aportes desde lo investigativo, la proyección social, la gestión del emprendimiento social, así como en la conceptualización práctica de la innovación social. Sin embargo, se han observado algunas debilidades en este programa que impiden la optimización de los resultados. El problema central identificado se relaciona con la escasa capacidad de crecimiento, entendido éste por el bajo volumen de la participación de docentes y estudiantes que pueden hacer parte de esta experiencia, junto con la estructura básica de gestión con la que se cuenta.

En vista de lo anterior, se desarrolló un ejercicio investigativo que permitió la construcción de una propuesta metodológica para gestionar la innovación social al interior de la universidad, a partir de ejercicios realizados en torno al emprendimiento, la investigación y la proyección social. A continuación se presentan los resultados más relevantes de la fase de diagnóstico, desde donde se analizaron los hallazgos más relevantes que han caracterizado a PIRI y la innovación social dentro de la AUNAR. Luego se presenta un modelo de gestión de Innovación Social, ISA, direccionado bajo los procesos misionales de AUNAR, dentro del Centro Regional de Emprendimiento y Empresarismo Autónoma de Nariño, CREAN, en la línea de investigación emprendimiento social.

\section{Marco teórico y métodos}

2.1 Programa de Internado Rural Interdisciplinario

El Programa de Internado Rural Interdisciplinario, PIRI, que ha sido liderado por la Universidad de la Frontera en la región de la Araucanía en Chile, en cabeza del departamento de salud pública, inició 
en el año de 1991 con un enfoque especial para trabajar desde el territorio como un escenario de aprendizaje y formación profesional. Este ejercicio académico se dio por una epidemia de hepatitis viral, momento en el cual el departamento de salud pública asume el reto social de contribuir a la construcción de una solución hacia esta problemática, desarrollando actividades como: Programas de prevención de la enfermedad, investigación, apoyo y reforzamiento de los centros de atención de salud y la generación de redes de relaciones colaborativas con las diversas instituciones y organizaciones locales, escenario que fue útil para el proceso de aprendizaje y formación profesional (Universidad de la Frontera, 1991).

El modelo educativo trascendió fronteras, incorporándose en Colombia a través de la Corporación Escuela del Sur, ES, entidad que ha desarrollado el programa en el municipio de Pasto desde el año 2014. Esta entidad aplica PIRI para trabajar con comunidades vulnerables, resolviendo problemáticas de tipo social, económico, educativo y de la salud; para ello se apoya en estudiantes de pregrado de algunas instituciones de educación superior de la ciudad, así como también en aliados gubernamentales y organizaciones internacionales que contribuyen metodológicamente para llevar a cabo el proceso (Morán, 2017).

La ES y AUNAR, en el marco del convenio pactaron desarrollar acciones conjuntas para fortalecer el PIRI, iniciando el trabajo desde julio de 2015, con la participación de estudiantes de las carreras de administración de empresas, diseño de modas, ingeniería mecánica y contaduría pública. Hasta el momento, el trabajo ha dado como resultado un análisis del entorno social, creando y trabajando líneas de acción, gestando sinergias tanto interdisciplinarias como interinstitucionales.

En AUNAR, PIRI ha generado importantes aportes desde lo investigativo, la proyección social, la gestión del emprendimiento social, junto con la conceptualización práctica de la innovación social. Gracias al programa, se han firmado conve- nios como el entablado con la Universidad de la Frontera, con quienes se ha abierto el camino para gestionar movilidades estudiantiles y docentes, para su incorporación a PIRI en el territorio chileno. A pesar de estos resultados, se ha observado la escasa capacidad de crecimiento del programa, caracterizada por el bajo volumen de docentes y estudiantes que pueden hacer parte de esta experiencia. Se espera que estas debilidades sean superadas con la propuesta metodológica que se presenta en las siguientes secciones.

\subsection{Metodología}

La investigación se ajusta a un enfoque mixto porque integra elementos tanto cuantitativos como cualitativos, a partir de los cuales se analiza la información permitiendo concluir sobre el proceso a través de unas estrategias que conllevan a cumplir con los objetivos trazados (Sampieri, Collado \& Lucio, 2004). Además, la investigación es de tipo descriptivo, pues narra características y propiedades de una situación específica, sin emplear juicios de valor y en procura de altos niveles de objetividad (Zuluaga-Duque, 2017).

La población objeto de estudio fueron 646 estudiantes matriculados durante 2017 en la Corporación Universitaria Autónoma de Nariño, AUNAR, quienes cursaban de sexto a décimo semestre en los diversos programas de formación profesional de la corporación. La muestra se ajustó a un nivel de confianza del $95 \%$, con un margen de error del $5 \%$, generando un total de 214 encuestas.

En las encuestas se formularon 23 preguntas, orientadas a la caracterización de la población, conocimiento y trabajos de innovación social dentro de la universidad, conocimiento del PIRI y sus beneficios, sugerencias para mejorar la implementación de programas de innovación social. También se identificaron las zonas rurales y urbanas donde les gustaría desarrollarlos.

La encuesta aplicada a los estudiantes de AUNAR buscaba indagar sobre su conocimiento acerca 
del Programa de Inserción Rural Interdisciplinaria, PIRI, así como sobre sus intereses en cuanto a la innovación social, y de qué manera les gustaría enrolarse en ellos. Lo anterior se complementó con una sesión de grupo con estudiantes que fueron participantes de la experiencia PIRI, junto con algunas entrevistas dirigidas a docentes y directivos tanto de AUNAR como de la Corporación Escuela del Sur, ES, entidad aliada en el desarrollo de PIRI.

\section{Resultados y discusión}

\subsection{Resultados cualitativos}

En el grupo focal participaron los estudiantes que han estado vinculados con PIRI, tanto de AUNAR como de las otras instituciones de educación superior participantes en la ciudad de Pasto, caracterizados por tener diversas formaciones profesionales y con personalidades distintas. A este grupo se le preguntó acerca de los aspectos positivos y negativos del programa PIRI, así como sobre las mejoras requeridas por PIRI para su articulación dentro de las universidades.

El grupo focal afirma que PIRI es una iniciativa que está dando resultados y que otorga una serie de herramientas, habilidades y destrezas que ayudan al profesional en el marco de su formación integral, especialmente por el enfoque social que les proporciona. Sin embargo, este ejercicio académico tiene algunas debilidades que deben superarse, como por ejemplo: poca articulación entre las instituciones que lideran el proceso, falta de claridad en los procesos para un buen desarrollo de PIRI, junto con la carencia de recursos y tiempos para la ejecución del programa.

En cuanto a las entrevistas, se realizaron las siguientes preguntas: ¿qué es lo bueno del programa PIRI para su universidad?; ¿qué encuentra de negativo en el desarrollo de PIRI, articulado entre su universidad y la Corporación Escuela del Sur?; ¿Qué mejoras considera que necesita PIRI para su articulación dentro de su universidad?; ¿ Que aporta el programa PIRI a los estudiantes y a su institución?. Los resultados obtenidos en las entrevistas se pueden sintetizar así:

PIRI es generador de impacto en el territorio, motivando al estudiante a tener una Inserción temprana de la aplicación de sus conocimientos y perfil profesional. Esto con el objetivo de generar desarrollo sostenible y sustentable, con equidad e inclusión social dentro de la comunidad.

La limitada disponibilidad de recursos estanca la ampliación del programa, causando un débil desarrollo de los participantes, junto con la sectorización de éstos por programa. Igualmente, falta un modelo de gestión que ayude a mejorar los procesos y acciones desarrolladas dentro del programa.

La mejora del programa debería contemplar su incorporación a las unidades académicas e investigativas de cada facultad, junto con el apoyo de instancias administrativas como el departamento de bienestar universitario y proyección social. Esto permitiría generar mayor visibilidad de PIRI en las instituciones participantes.

PIRI aporta y genera una serie de herramientas que ayudan a los estudiantes a fortalecer sus cualidades sociales, académicas e investigativas, cimentando sus conocimientos y ganando experiencia. Dentro de la universidad, el programa genera impacto social positivo, producción científica e internacionalización de los estudiantes.

En cuanto a los puntos clave de PIRI, los entrevistados resaltaron los siguientes: La participación de estudiantes y docentes como agentes de interdisciplinariedad, conocimiento y creatividad, generando procesos que permiten sensibilizar a los estudiantes; la participación activa de la comunidad como base para la realización de proyectos y el cumplimiento de los objetivos planteados cada semestre; formar estudiantes con enfoque participativo e incluyente, que permitan generar procesos de capacitación y concientización permanente. 
En síntesis, las entrevistas permiten destacar los siguientes aspectos:

PIRI aporta a la academia un mecanismo concreto de relacionamiento y cooperación con el territorio. Además, facilita una interacción directa de los estudiantes con la sociedad, permitiéndoles relacionar los conocimientos técnicos adquiridos hacia una contribución al desarrollo regional de forma más práctica, interdisciplinar y transversal (AUNAR, 2013).

Un alcance limitado por la disponibilidad de recursos, hace que el programa se enfoque en un solo lugar para implementar acciones de innovación social, situación que limita la vinculación de más estudiantes por universidad y programa académico. Para la articulación de PIRI, una de las mejoras importantes que se puede implementar es tener mayores espacios de intercambio, retroalimentación y cooperación directa con las universidades vinculadas (Morán, 2016).

PIRI aporta una amplia noción de realidad, apego e identidad con el territorio y la cultura, valoración de lo propio y reconocimiento del entorno. Adicionalmente, la reflexividad, capacidad de análisis, comprensión de las situaciones sociales, compañerismo, apertura a otras disciplinas, habilidades comunicacionales, trabajo en equipo, liderazgo y aporte a construcción de paz son competencias que se fortalecen con la metodología del programa. En este sentido, uno de los puntos clave para el desarrollo de programas como PIRI es la comunicación y relacionamiento entre comunidades, líderes, estudiantes, docentes y organizaciones de base social comunitaria (Pérez \& Castaño, 2016).

Lo anterior indica que es de suma importancia direccionar los procesos realizados en el PIRI de forma más sistemática, acorde con el objetivo de incrementar la gestión dentro del territorio. Esto permitirá fortalecer su calidad y ampliar la cobertura, facilitando la participación de una mayor cantidad de estudiantes e instancias administrativas dentro del acuerdo interinstitucional. Igualmente, es necesario contar con una estructura que direccione cada una de las actividades lideradas por las universidades, lo cual facilitaría de manera práctica, sencilla y apropiada, hacer un seguimiento oportuno, orientado a elevar el impacto social de las acciones. Esto es clave en los programas de responsabilidad social empresarial, más aún tratándose de instituciones de educación superior (Vergara-Arrieta \& Carbal-Herrera, 2016; Balza-Franco \& Cardona-Arbeláez, 2015).

\subsection{Resultados cuantitativos}

En este apartado se encontró que el 43,5\% de los encuestados entiende por innovación social al proceso de: diseñar, desarrollar y generar nuevas ideas, que tienen como objetivo cubrir necesidades de la sociedad. Igualmente, el 53,3\% afirma que la mejor manera de impulsar la innovación social es a través de programas que trabajen este tema. Además, el $76,6 \%$ de los encuestados afirma que la innovación social debe trabajarse en las zonas rurales y urbanas, y $11,2 \%$ en zonas afectadas por el conflicto armado, prefiriendo trabajar con población infantil $(49,5 \%)$ y madres cabeza de familia $(21,5 \%)$.

Adicionalmente, $71 \%$ de la población encuestada no ha participado en programas ni actividades relacionadas con innovación social y un 15,9\% afirma que ha participado algunas veces. Sin embargo, al 57,3\% le gustaría algunas veces trabajar en programas de innovación social y un $26,3 \%$ estaría dispuesto siempre a trabajar en ellas.

El $26,5 \%$ de la población considera que al ejercicio PIRI, para ser un modelo exitoso de innovación social, le faltan más personas que participen. Además, un $24,8 \%$ sugieren que le falta más socialización de beneficios, ampliar los lugares para su desarrollo, más personas participantes y una estructura que lo identifique.

El diagnóstico anterior permite establecer las siguientes necesidades: potencializar la comunicación interna y externa de PIRl; difundir las acciones 
realizadas dentro de las instancias académicas y administrativas; articulación del programa a las dependencias administrativas, académicas e investigativas; dar continuidad a las acciones y los procesos; aumentar la cobertura y desarrollo de PIRI en zonas rurales y urbanas; incrementar la producción científica dentro de la universidad; potencializar la participación de la comunidad estudiantil en las modalidades ofertadas por PIRI.

\subsection{Matrices de análisis}

Para el análisis de los resultados se hizo uso de la Matriz de Evaluación de Factores Internos, MEFI, y la Matriz de Evaluación de Factores Externos, MEFE. Estas herramientas permiten identificar variables de mayor peso, que ayudan al cruce de la matriz de Fortalezas, Oportunidades, Debilidades y Amenazas, FODA, contribuyendo a identificar las potencialidades, desafíos, riesgos y limitantes del programa, para construir objetivos y estrategias que ayuden a su mejoramiento.

Luego de aplicar las matrices, se estableció que al analizar los factores internos, el peso ponderado fue de 2,55. Esto se puede interpretar como que PIRI, dentro del desarrollo de la AUNAR, está fundamentado en fortalezas de gran efectividad, generando una serie de beneficios que hacen tangible la innovación social, involucrando a la academia con la sociedad y fortaleciendo las competencias de los participantes, de manera que se contribuye con: la producción científica, el desarrollo con equidad social y sostenible. Sin embargo, se requiere mitigar las debilidades que limitan su crecimiento y proyección, tanto dentro de la universidad como en el territorio.

Entre las debilidades que deben ser mitigadas está la escasa disponibilidad de recursos financieros, que como efecto directo limita los beneficios que genera el programa PIRI. Esto se podría mitigar al articular las dependencias administrativas y académicas, con el fin de que se optimicen los recursos. Otra debilidad identificada es la débil estructura organizacional que direccione cada uno de los procesos con claridad, dentro del desarrollo de las actividades lideradas por la universidad, lo cual ocasiona desaprovechamiento de los recursos y poca optimización de los mismos.

El total del peso ponderado arrojado por el análisis de los factores externos es de 2,52. Esto demuestra que PIRI, dentro del desarrollo con AUNAR, cuenta con oportunidades que pueden ayudar al programa a ampliarse desde la misma universidad. En este sentido, existe la facilidad de concretar sinergias que impulsan redes de cooperación interinstitucional, convertirse en facilitadores o entes consultores ante la mejora del programa, fortaleciendo el desarrollo y atenuando las amenazas que lo rodean.

En cuanto a las amenazas, se encontró que la falta de compromisos territoriales por parte de las entidades gubernamentales regionales, puede causar incertidumbre en la comunidad donde se desarroIlan los procesos. Esta situación se podría mitigar aprovechando la oportunidad de realizar sinergias con entidades que generan confianza para el acompañamiento de los procesos, como el Centro de Innovación Social del Departamento de Nariño (CISNA), así como el Programa de las Naciones Unidas (PNUD), lo cual a su vez genera el desafío de articular la cooperación interinstitucional dentro de PIRI.

Del anterior análisis se realizó un cruce de factores internos y externos, mediante un FODA de cuatro celdas, identificando 10 factores claves de éxito para mejorar la gestión universitaria en innovación social dentro de la institución; estos se han sintetizado en la tabla 1. 
Irina Margarita Jurado-Paz

Michael Alexander Morán-Vallejo

Tabla 1. Potencialidades y desafíos para mejorar la gestión universitaria. 


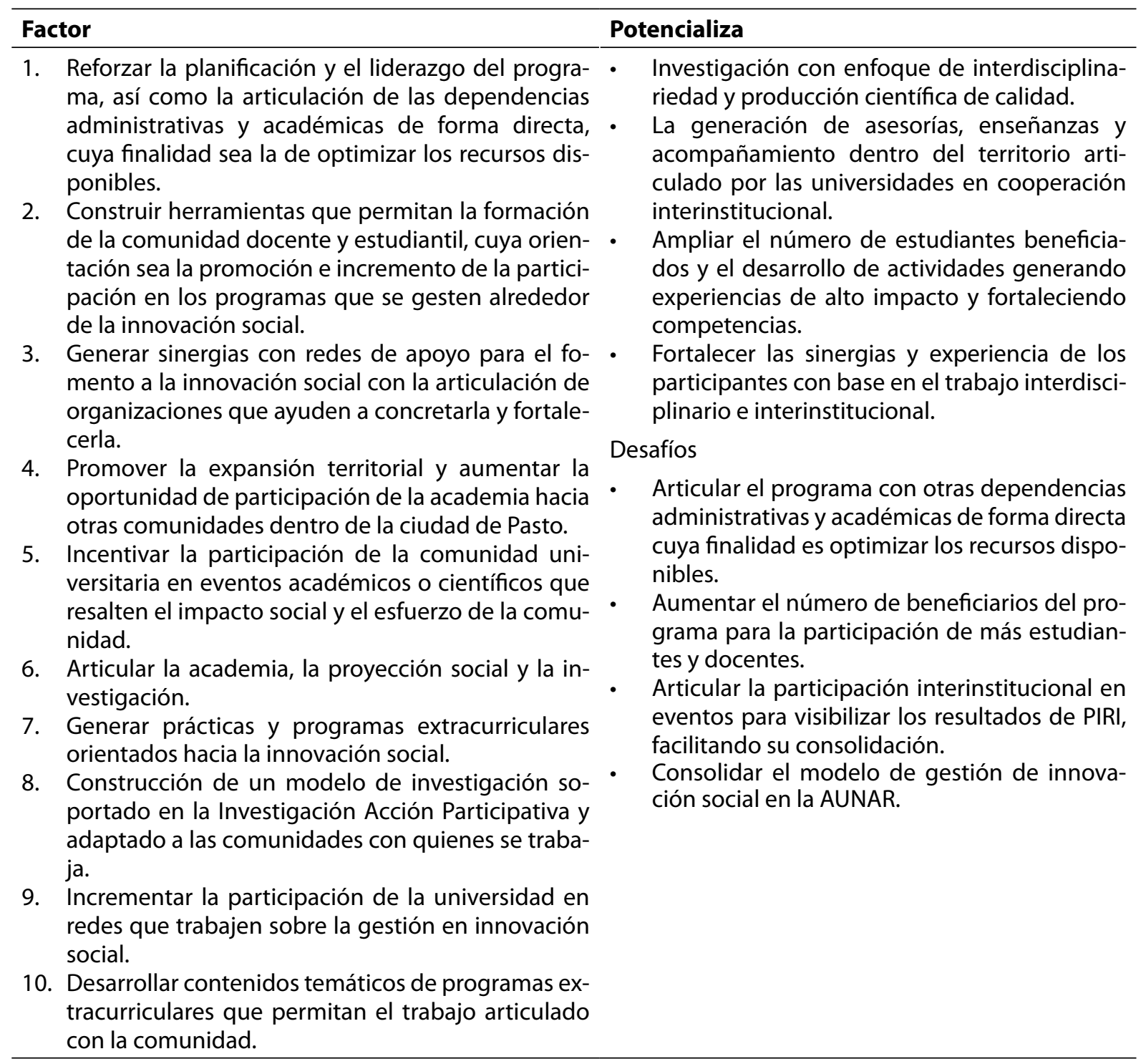

\subsection{Modelo de gestión propuesto}

A partir del análisis anterior, se procede a la construcción de los objetivos, tanto generales como específicos, así como de las estrategias previstas para dar cumplimiento a la propuesta de mejora para la gestión universitaria en innovación social. Estos elementos se muestran en la tabla 2. 
Tabla 2. Objetivos del modelo de gestión.

Objetivo general: Mejorar el desarrollo de la innovación social dentro de la Corporación Universitaria Autónoma de Nariño (AUNAR).

\begin{tabular}{|c|c|}
\hline Objetivo & jias \\
\hline $\begin{array}{l}\text { 1. Reforzar la planificación a partir de modelos de } \\
\text { liderazgo, la articulación de las dependencias } \\
\text { administrativas y académicas, con la finalidad } \\
\text { de optimizar los recursos disponibles. } \\
\text { 2. Construir herramientas que permitan la forma- } \\
\text { ción de la comunidad docente y estudiantil, } \\
\text { cuya orientación sea la promoción e incremen- } \\
\text { to de la participación en los programas que se } \\
\text { gesten alrededor de la innovación social. } \\
\text { 3. Generar sinergias con redes de apoyo para el } \\
\text { fomento de la innovación social con diferentes } \\
\text { organizaciones que contribuyan a fortalecerla. } \\
\text { 4. Promover la expansión territorial y aumentar la } \\
\text { oportunidad de participación de la academia } \\
\text { con otras comunidades dentro del territorio. } \\
\text { 5. Incentivar la participación de la comunidad } \\
\text { universitaria en eventos académicos o cientíi- } \\
\text { cos que resalten el impacto social y el esfuerzo } \\
\text { de la comunidad. }\end{array}$ & $\begin{array}{l}\text { Articulación de la academia, proyección social e in- } \\
\text { vestigación (cumple con objetivo estratégico 1). } \\
\text { Generación de prácticas y programas extracurricu- } \\
\text { lares orientados hacia la innovación social (cumple } \\
\text { con objetivo estratégico 2). } \\
\text { Construcción de un modelo de investigación so- } \\
\text { portado en la Investigación Acción Participativa y } \\
\text { adaptado a las comunidades con quien se trabaja } \\
\text { (cumple con objetivos estratégicos } 5 \text { y 2). }\end{array}$ \\
\hline
\end{tabular}

Con el desarrollo de este tipo de modelo de gestión universitaria en innovación social, se fortalecen las habilidades, actitudes, aptitudes y perspectivas de los estudiantes, mediante el aprendizaje que ayuda abordar las problemáticas sociales y el manejo adecuado, tanto de las herramientas como de los métodos, para operar eficazmente. En este sentido, se fortalecen las competencias de efectividad personal o ciudadanas a ser adquiridas por los estudiantes, que se resumen de la siguiente manera:

Incrementar el conocimiento por parte de los estudiantes sobre las necesidades de la comunidad.

Formar estudiantes íntegros que conduzcan a obrar, sentir y respetar en todo momento, con valores morales, las buenas costumbres y prácticas profesionales, respetando las políticas organizacionales de la comunidad.

Aumentar la creatividad, profundizándo la capacidad del estudiante en modificar las cosas, par- tiendo de formas o situaciones no pensadas. Tiene como finalidad crear soluciones nuevas a diferentes problemas o situaciones, requeridas en el trabajo con comunidades.

En síntesis, el modelo propuesto busca que haya una interacción de la academia, la investigación, la proyección social de la universidad y el trabajo con comunidad. Este último se centra en las capacidades y la sinergia entre los estudiantes y los miembros de un grupo social determinado, desde donde puedan gestarse transformaciones que contribuyan a la solución de dificultades específicas y empodere a los habitantes para que ellos mismos generen los posteriores controles o correctivos, que finalicen con mejoras aún más significativas para todos (Valle Chirinos-Araque \& Pérez-Peralta, 2016).

Este modelo, a diferencia del implementado por otros autores $u$ otras instituciones univer- 
sitarias, fortalece la interdisciplinariedad, se enriquece del trabajo en equipo y potencializa las capacidades individuales de sus participantes hacia el cumplimiento de un objetivo común, además de beneficiarse con los aportes propios de la innovación social, evitando "compadecer" a una población determinada o desarrollando modelos de acompañamiento comunitario bajo esquemas asistencialistas.

\section{Conclusiones}

Los resultados indican que en la población estudiantil analizada, existe un gran interés de participar en los programas sociales que se realizan con un enfoque de investigación e innovación social. En este sentido, se propusieron estrategias que buscan potencializar la difusión y la articulación de instancias administrativas, académicas e investigativas hacia el cumplimiento del proyecto institucional y plan de desarrollo, de la universidad estudiada.

Lo anterior se puede lograr a través de un modelo de gestión universitaria en innovación social, que fungiría como un mecanismo concreto de relacionamiento y cooperación con el territorio. Esto facilitará la interacción directa entre los estudiantes y la sociedad, permitiéndoles enfocar los conocimientos adquiridos de forma más práctica, interdisciplinar y transversal. Esto último permitirá la articulación del emprendimiento, la investigación y la proyección social hacia el cumplimiento de un objetivo común.

Finalmente, para que un programa de innovación social ejecutado desde las instituciones de educación superior, cumpla con su cometido, se deben contemplar factores cuantitativos y cualitativos, tales como: potencializar la comunicación interna y externa; articular el programa con las dependencias administrativas, académicas e investigativas. También es importante dar continuidad a las acciones y los procesos, aumentando la cobertura y desarrollo de programas como PIRI en zonas rurales y urbanas. Esto posibilitará incrementar la pro- ducción científica para beneficio de la comunidad académica y de la universidad.

\section{Referencias}

Balza-Franco, V. B., \& Cardona-Arbeláez, D. (2015). La Responsabilidad Social Empresarial Y La Lucha Contra La Pobreza. Saber, ciencia y libertad, 10 (1), 115-124. doi: http://dx.doi.org/10.22525/sabcliber.2015v10n1.1151

Cardona-Arbeláez, D., Alzate-Alvarán, J. C., \& Lora-Guzmán, H. (2018). Estrategias para la gestión del talento humano en las asociaciones de yuqueros adscritas a Colfeyuca en Sucre, Colombia. Revista de Investigación, Desarrollo e Innovación, 9 (1), 9-18. doi: https://doi.org/10.19053/20278306. v9.n1.2018.8501

Comisión de las Comunidades Europeas. (2001). El libro verde: Fomentar un marco europeo para la responsabilidad social de las empresas.

Corporación Universitaria Autónoma de Nariño, AUNAR. (2013). Plan de Desarrollo Institucional 2013-2023. Pasto, Colombia.

Del Río-Cortina, J., Cardona-Arbeláez, D., \& Guacarí-Villalba, A. (2017). Responsabilidad social empresarial y construcción de la marca: una nueva mirada a las estrategias de gestión. Revista de Investigación, Desarrollo e Innovación, 8 (1), 49-60. doi: https://doi.org/10.19053/20278306. v8.n1.2017.7370

López-de Parra, L., Polanco-Perdomo, V., \& Correa-Cruz, L. (2017). Mirada a las investigaciones sobre formación investigativa en la universidad latinoamericana: estado del arte 2010 a 2017. Revista de Investigación, Desarrollo e Innovación, 8 (1), 77-95. doi: https://doi.org/10.19053/20278306. v8.n1.2017.7371

Morán, M. (2016). Propuesta para la creación de la unidad de emprendimiento en la Institución Educa- 
tiva la Victoria del municipio de Ipiales para el año 2017. Pasto, Colombia: AUNAR.

Morán, M. (2017). Plan de mejoramiento para el convenio suscrito entre la Corporación Universitaria Autónoma de Nariño y la Corporación Escuela del Sur, en marco del acuerdo de cooperación interinstitucional dentro del Programa de Internado Rural Interdisciplinario -PIRI- en el municipio de Pasto para el año 2018. Pasto, Colombia: Corporación Universitaria Autónoma de Nariño.

Pérez, S., \& Castaño, R. (2016). Funciones de la Universidad en el siglo XXI: humanística, básica e integral. Revista Electrónica Interuniversitaria de Formación del Profesorado, 19 (1), 191囚199. Recuperado de: http://revistas.um.es/reifop/article/ view/202451

Rodríguez, A., \& Alvarado, H. (2008). Claves de la innovación social en América latina y el Caribe. Santiago de Chile, Chile: CEPAL.

Sampieri, R. H., Collado, C. F., \& Lucio, P. B. (2004). Metodología de la investigación. Ciudad de México, México: McGrawHill.

Universidad de la Frontera. (1991). Programa de Internado Rural Interdisciplinario (P.I.R.I). Recuperado de: http://piri.ufro.cl/index.php/piri/historia

Valle Chirinos-Araque, Y., \& Pérez-Peralta, C. M. (2016). La Responsabilidad Social Universitaria: emprendimiento sostenible como impacto de intervención en comunidades vulnerables. Revista EAN, (81), 91-110. doi: https://dx.doi. org/10.21158/01208160.n81.2016.1560

Vergara-Arrieta, J., \& Carbal-Herrera, A. (2014). Diseño de un sistema de gestión en responsabilidad social empresarial para pequeños hoteles en la ciudad de Cartagena. Saber, Ciencia y Libertad, 9 (2), 91-108. doi: http://dx.doi.org/10.22525/sabcliber.2014v9n2.91108
Zuluaga-Duque, J. F. (2017). Relación entre conocimientos, saberes y valores: un afán por legitimar los saberes más allá de las ciencias. Revista de Investigación, Desarrollo e Innovación, 8 (1), 61-76. doi: 10.19053/20278306.v8.n1.2017.5973 
Gestión universitaria de la innovación social promovida desde espacios académicos relacionados con el emprendimiento, la investigación y la proyección social 